\section{The regeneration game}

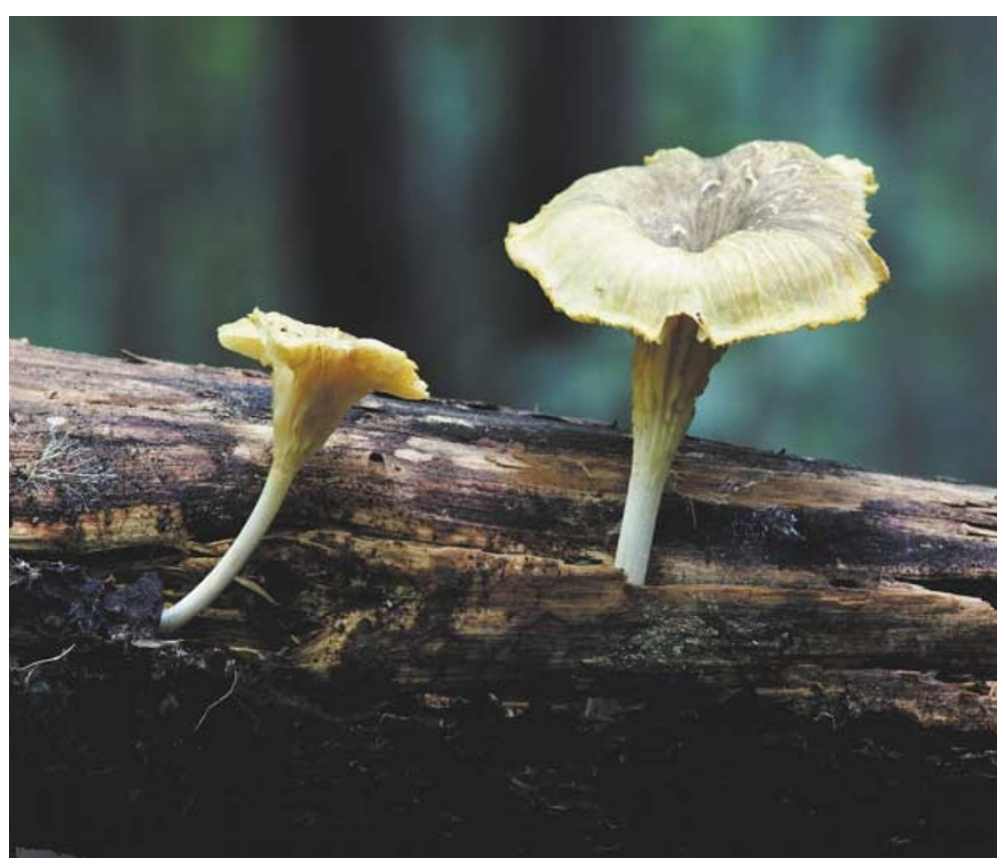

The poor regenerative capacity of axons in the central nervous system (CNS) is still a serious obstacle to CNS repair. However, two new studies published in Neuron, by Qiu et al. and Neumann et al., indicate that a solution to this problem might come from studying how dorsal root ganglion (DRG) neurons respond to peripheral damage.

The primary sensory neurons of the DRG have axonal branches in both the peripheral nervous system and the spinal cord. But whereas the peripheral branch can regenerate after injury, the central branch cannot. Intriguingly, however, if the peripheral branch is lesioned first, the regenerative capacity of the central branch is increased considerably. The authors of these studies reasoned that if they could identify the signalling pathways that are activated by this socalled 'conditioning lesion', they might be able to mimic its effects without causing further damage.

It was previously shown that raising the levels of cyclic AMP signalling in CNS neurons allows them to grow on myelin, which normally inhibits their growth. Qiu et al. showed that cAMP levels were raised in DRG neurons in response to a peripheral lesion, so they asked whether cAMP signalling was required for the conditioning effect. They found that, in the presence of a cAMP inhibitor, a peripheral lesion could no longer stimulate central axonal growth, providing strong evidence that the conditioning response is mediated by cAMP signalling.

Could cAMP signalling mimic the effects of a peripheral lesion on central axonal regeneration? Both teams increased the level of cAMP signalling in primary sensory neurons by injecting a cAMP analogue into the DRG, where the cell bodies of these neurons are located. They found that this treatment did indeed cause central axons to regenerate in the absence of a peripheral lesion. In addition, Neumann et al. showed that this treatment has a

\title{
From single lane to superhighway
}

It was initially hard to believe that, despite the large number of vesicles docked at any central synapse, transmitter release was monovesicular - an action potential released only one vesicle from a given active zone. But this idea has slowly gained such strong support, that it is now seldom disputed. Oertner et al. have taken a new look at this issue, using optical methods to measure release at a single synapse. Their data show that, contrary to the monovesicular model, several vesicles can simultaneously release their content from single active zones.

Instead of measuring synaptic responses using electrophysiological methods, Oertner et al. opted for visualizing postsynaptic NMDA ( $N$-methyl-Daspartate)-receptor-dependent calcium transients. Glutamate release increased intracellular calcium at single spines, allowing the authors to distinguish trials in which release occurred (when there was a calcium transient) - successes - from those in which there was no vesicle fusion (no calcium signal) - failures. They measured how the transients changed under conditions that affect release probability, such as paired-pulse facilitation (PPF). In PPF, two action potentials that arrive in quick succession lead to a larger synaptic response after the second stimulus. If release were monovesicular, then PPF should not lead to further increases in the amplitude of a calcium transient, because the amount of glutamate that is released by a single vesicle is relatively homogeneous. However, Oertner et al. found that calcium increases in response to the second stimulus were significantly larger than those evoked by single action potentials, implying that PPF causes more glutamate to be released at single synapses. The simplest explanation for this finding is that transmitter is released from more than one vesicle. Indeed, the data agree with the predictions of the classical binomial model for multiple release sites, and the authors propose the existence of five independent release sites per active zone.

Measuring postsynaptic responses with this optical method arguably provides the most direct evidence for multivesicular release at a single central synapse. Clearly, the ability of a single active zone to release more than one vesicle at a time markedly increases the dynamic range of the synapse. Whether long-term plastic changes in transmission make use of this capacity is an intriguing question.

Juan Carlos López

(2) References and links ORIGINAL RESEARCH PAPER Oertner, T. G. et al. Facilitation at single synapses probed with optical quantal analysis. Nature Neurosci. 5, 657-664 (2002) FURTHER READING Atwood, H. L \& Karunanithi, S. Diversification of synaptic strength: presynaptic elements. Nature Rev. Neurosci. 3, 497-516 (2002) WEB SITES

Karel Svoboda's laboratory: http://www.cshl.org/labs/svoboda/ 
dual effect - it not only helps the axon to overcome the inhibitory effects of myelin, but also increases its intrinsic capacity for growth.

So, the conditioning effect of a peripheral lesion can be reproduced by stimulating cAMP signalling in the DRG. The fact that this intervention can be carried out at the level of the cell body means that it is not necessary to inflict further trauma on the site of injury, raising the possibility that it could lead to a viable clinical treatment for spinal cord damage. At a more fundamental level, it will also be interesting to elucidate the molecular basis of the asymmetrical response of DRG neuronal processes to injury.

Heather Wood

(2) References and links ORIGINAL RESEARCH PAPER Neumann, $S$. et al. Regeneration of sensory axons within the injured spinal cord induced by intraganglionic cAMP elevation. Neuron 34, 885-893 (2002) | Qiu, J. et al. Spinal axon regeneration induced by elevation of cyclic AMP. Neuron 34, 895-903 (2002)

FURTHER READING Filbin, M. T. The benefits of adding insult to injury. Neuron 23, 2-4 (1999)

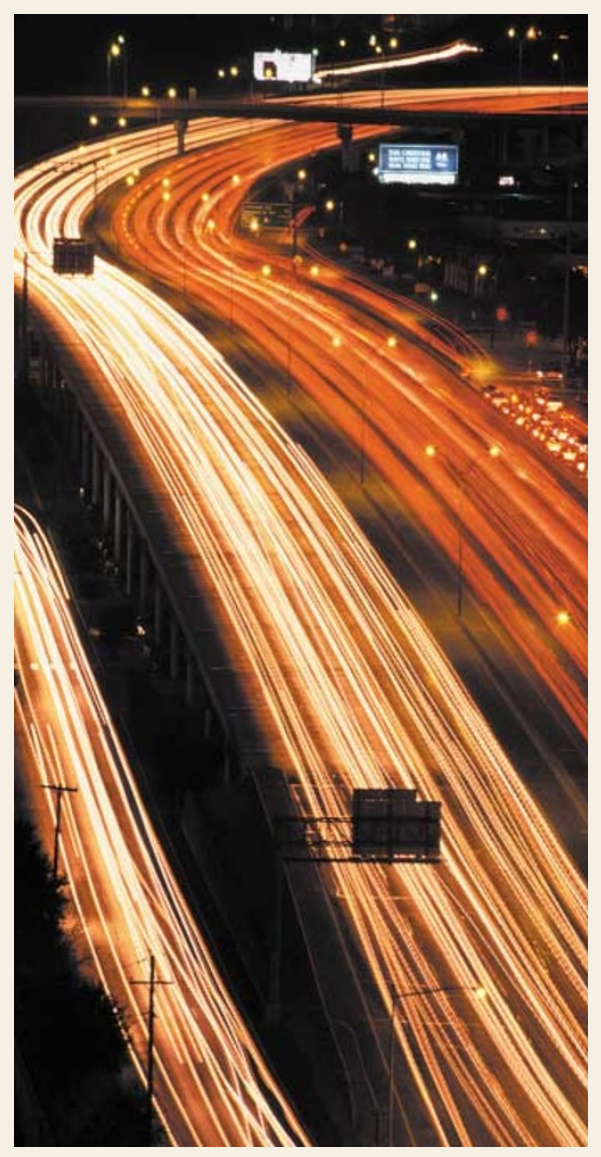

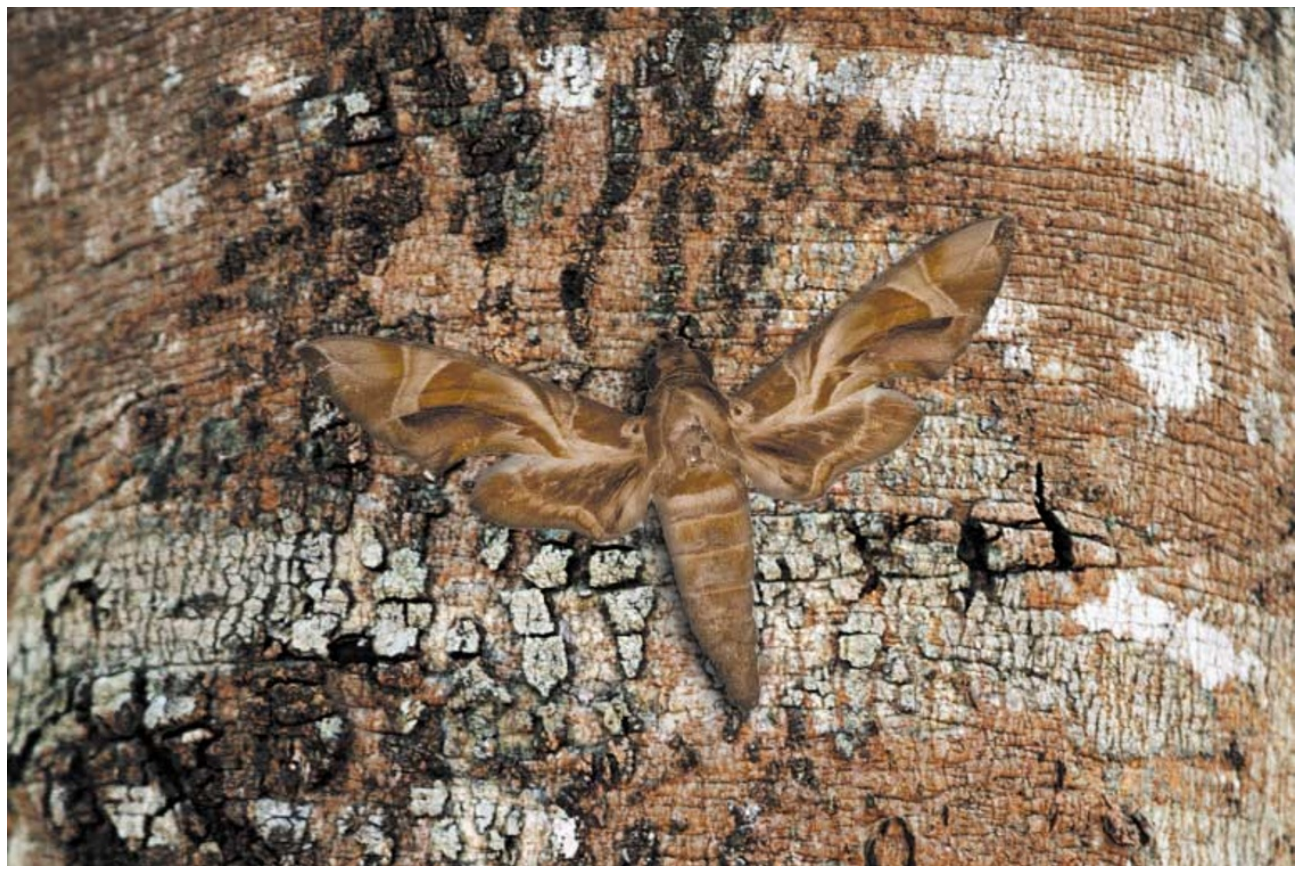

VISUAL ATTENTION

\section{Now you see it...}

It's surprising just how little we actually see when we aren't paying attention. Despite the subjective impression that we are taking in the entire visual field, most studies find that we remain unaware of most of the details of areas of space on which we are not focusing. It is generally thought that only 'primitive' visual features - colour or orientation, for example - can be picked out pre-attentively, because these features are processed very early in the visual system. But two new studies cast doubt on this assumption.

Both of the studies used natural scenes, rather than artificial psychophysical stimuli, to test visual processing. Li et al. asked subjects to attend to a task, presented in the centre of the visual field, in which they had to discriminate between $\mathrm{T}$ and $\mathrm{L}$ shapes. At the same time, they were asked to detect whether a scene presented in the periphery of the visual field contained an animal.

Conventional wisdom would predict that this second task would be very difficult to do when attentional resources were being used up by the central letter discrimination. But performance on the animal detection part of the task was just as good when it was presented along with the letter task as when it was done on its own, suggesting that subjects can detect and categorize the complex sets of visual features that make up an animal even when they don't devote their attention to it.
The other study, carried out by Rousselet et al., also required subjects to detect whether an animal was present in a scene. This time, however, the researchers presented subjects with either one or two pictures, one on each side of the visual field, while the subjects fixed their vision on a central point. Subjects could correctly detect whether an animal was present just as quickly for two pictures as they could for one. This indicates that the images were categorized rapidly and in parallel, a claim that was confirmed by simultaneously recording electrical activity of the brain.

Both of these studies raise many questions about visual processing. For example, it is unclear whether the pictures in the second study were processed separately by the two

hemispheres of the brain. Would the results have been the same if the two pictures had been presented above and below the fixation point, instead of to the left and right? And do we process complex features of unattended visual scenes in the same way even if we aren't trying to detect a specific type of stimulus (in this case, an animal)? Nonetheless, it is clear that the gating of visual information by attention is much less simple than we thought, and that 'early' processing can include processes that occur at high levels of the visual system.

Rachel Jones

(2) References and links ORIGINAL RESEARCH PAPER Li, F. F. et al. Rapid natural scene categorization in the near absence of attention. Proc. Natl Acad. Sci. USA 20 June 2002 (doi:10.1073/pnas.092277599) | Rousselet, G. A. et al. Parallel processing in high-level categorization of natural images. Nature Neurosci. 5, 629-630 (2002)

FURTHER READING Kanwisher, N. \& Wojciulik, E. Visual attention: insights from brain imaging. Nature Rev. Neurosci. 1, 91-100 (2000) 